\title{
Superconductivity Controlled by Polarization in Field-Effect Devices of Confined Geometry
}

\author{
Natalia Pavlenko ${ }^{1,2} *$ and Franz Schwabl ${ }^{1}$ \\ ${ }^{1}$ Institut für Theoretische Physik T34, Physik-Department der TU München, \\ James-Franck-Strasse, D-85747 Garching b. München, Germany \\ ${ }^{2}$ Institute of Physics, University of Augsburg, 86135 Augsburg, Germany
}

\begin{abstract}
We propose a concept for superconducting electric field-effect devices based on superconducting films sandwiched between ferroelectric layers. We provide theoretical calculations that indicate how the field effect in these devices could be amplified, which can be experimentally probed even at the current stage of film fabrication techniques.

PACS numbers: 74.80.-g,74.80.Dm,77.80.-e,79.60.Jv
\end{abstract}

The electric field effect in superconductors attracts considerable attention in science and technology [1]. The external field can modulate the charge density and resistance, and control a reversible superconductor-insulator switching behavior, which plays a key role in superconducting field-effect transistors (SuFETs) based on dielectric or ferroelectric polarisation. Especially in hightemperature superconducting oxides, where the low carrier density $\sim 10^{21} \mathrm{~cm}^{3}$ yields larger electric penetration depths $\lambda_{T F} \sim 1 \mathrm{~nm}$, a switch of the ferroelectric polarization is found to produce $\sim 10 \%$ modulation in the carrier density $n$ at the interface 2]. The observed changes in $n$ and in the superfluid density $n_{S}$ are shown to be the key factor responsible for a several $\mathrm{K}$ shift in the superconducting transition temperature $T_{c}$ 2, 3], in paricular in the underdoping region where $T_{c}$ is proportional to $n_{S}(0)$ [4]. In the superconducting films of a thickness $\gg \lambda_{T F}$, the shift of $T_{c}$ due to the charge modulation in the interface region of about $1 \mathrm{~nm}$ thickness is shunted. Thus, it is especially advantageous for SuFETs to use ultrathin superconducting channels of a few nm thickness $[3,5]$. To make such SuFETs suitable for technological applications, the achievement of $T_{c}$ shifts beyond the range $5-10 \mathrm{~K}$ is required. This needs the mechanisms of a possibly stronger charge density modulation by the gate polarization in the range $10-30 \mu \mathrm{C} \mathrm{cm}^{-2}$ which is currently achievable in ferroelectric oxides $\mathrm{Pb}(\mathrm{Zr}, \mathrm{Ti}) \mathrm{O}_{3}(\mathrm{PZT})$ and (Ba,Sr) $\mathrm{TiO}_{3}$ (BSTO).

Recently, theoretical studies of superconductingferroelectric (S-FE) multilayers [6] have shown that the modulation of charge in the ultrathin superconducting films sandwiched between the ferroelectric layers is much stronger than in ferroelectric-superconductor bilayers typically exploited for SuFETs. In a sandwich-like $\mathrm{FE}_{1}-\mathrm{S}-\mathrm{FE}_{2}$ heterostructure (Fig. 1(a)), the polarization $P_{2}$ in the second gate $\mathrm{FE}_{2}$ pushes an extra charge from the interface $\mathrm{S}-\mathrm{FE}_{2}$ into the accumulation region at the interface $\mathrm{FE}_{1}-\mathrm{S}$ of the $\mathrm{S}-$ film. For high- $T_{c}$ cuprates as the most compatible candidates for the sandwiches, the redistribution of carriers between the interfaces driven by $P_{1}$ and $P_{2}$ could require S-films of $2-3$ unit cell thick- ness. In such films, the charge redistribution between $\mathrm{CuO}_{2}$-planes can occur via the interplanar tunneling of Cooper pairs which provides not only the way for the charge modulation, but also enhances the local $T_{c}(l o c)$ in the accumulation region and thereby allows achievement of higher $T_{c}$ in the entire S-film. For possible realizations of SuFETs based on FE-S-FE sandwiches, the question that needs to be addressed is how the superconducting properties can be controlled by the voltages in FE-gates which is the subject of present studies.

Here we consider a superconducting film containing $L_{S}=2$ or 3 infinite 2D-planes, as shown in Fig. 1(a) for $L_{S}=2$. The superconductivity in each plane is described by a BCS-like model with an effective pairing potential $V^{0}$ (except that the energy cutoff is determined by the electron bandwidth). These planes are weakly connected by the interplanar Cooper pair tunneling with the tunneling energy $t_{\perp} / t=0.05-0.1(t \approx 0.1 \mathrm{eV}$ for high$\mathrm{T}_{c}$ cuprates is the nearest neighbor hopping energy on a square lattice, which sets the energy scale). The S-film is sandwiched between the FE-layers of a thickness given by the number $L_{F}$ of unit cells in $z$-direction perpendicular to the interfaces. In SuFETs, the charge redistribution in the S-film can be achieved by reorienting the polarizations $P_{1}$ and $P_{2}$ in the layers $\mathrm{FE}_{1}$ and $\mathrm{FE}_{2}$ perpendicular to the interfaces by the gate electric field $E_{g}^{1}$ and $E_{g}^{2}$. Hence, we focus essentially on the two possible orientations of ferroelectric dipoles (one of them is shown in Fig. 1(a)), representing them by two values $\pm 1 / 2$ of a pseudospin (dipole) operator. To describe by this pseudospin formalism the nonzero spontaneous polarization in each FE-layer due to ion displacements below the Curie temperature, we employ an Ising model with the dipole-dipole interaction energy $J_{F}$ taken into account in addition to the interactions $-E_{g}^{1} \cdot P_{1}$ and $-E_{g}^{2} \cdot P_{2}$ with the gate fields [7]. The screening of the polarization at the interfaces by the charge in the S-film is described by the electrostatic charge- ferroelectric dipole interaction $\gamma=\frac{e}{\Delta_{S F}^{2}} \cdot d_{F E}$. Here $d_{F E}$ is the magnitude of the dipole in each FE-unit cell and $\Delta_{S F}$ is the distance between the nearest FE-unit cell and S-film. In our analysis, the in- 
terface energy $\gamma$ ranges from zero (isolated S-film) to $\sim t$, which should lie in a typical range of the charge-dipole interactions at the contacts with ferroelectric BSTO(PZT)layers of $\sim 100-300 \AA$ thickness where the polarization $\lesssim 25 \mu \mathrm{C} \mathrm{cm}^{-2}$ is suppressed due to strong depolarization fields 8]. We study the system far below the Curie temperature, treating the ferroelectric polarization in the mean-field approach 9, 10]. Then the superconducting gaps and the polarization profiles are calculated selfconsistently by the numerical minimization of the free energy of the system [6].

We assume that the charge described by the electron concentration $n$ per the unit cell in S-film, is already injected (resulting in the superconducting state due to the effective attraction), and focus on the question of how the superconductivity can be affected by the gate polarization. When injected into the film, the charge screens the interface polarization $P_{1}$ and $P_{2}$. The resulting interplanar charge redistribution is described by the difference of the carrier density in the boundary planes $\Delta n=n_{1}-n_{L_{S}} \approx \frac{\gamma}{4 t}\left(P_{1}+P_{2}\right)$. The parallel polarization $\left(P_{1}=P_{2}=P>0\right)$ leads to $\Delta n>0$, to the accumulation of electrons in plane $i_{S}=1$ and their depletion in plane $i_{S}=L_{S}$. The polarization $P_{2}$ in the second gate acts as an additional driving force pushing the charge from the interface $\mathrm{S}-\mathrm{FE}_{2}$ to the plane $i_{S}=1$ and thus providing stronger charge modulation in sandwiches.

The accumulation plane $i_{S}=1$ has higher local $T_{c}^{1}(l o c)$ and leads to increase of $T_{c}$ in the entire $\mathrm{S}$-film due to the interplanar coupling [6]. For the hole carriers in the underdoped region of high- $T_{c}$ cuprates, the higher local $T_{c}(l o c)$ is achieved in the electron depletion (hole accumulation) plane $i_{S}=L_{S}$ and in the following discussion one should consider the hole instead of electron accumulation. As compared to the isolated film (case $\gamma=0$ ), the $P$-induced increase of $T_{c}$ is clearly seen in Fig. 11(b) for the parallel FE-gate polarizations $\left(P_{1}=P_{2}\right)$. The $T_{c}$ increase in this state does not depend on the sign of $P_{1}$ and $P_{2}$, since their simultaneous switch merely leads to the switch between the location of the accumulation and depletion region without affecting the final $T_{c}$ [6]. In contrast to the state with parallel polarization, the antiparallel polarization in the FE-gates $\left(P_{1}=-P_{2}>0\right)$ results in a symmetric electron accumulation at both interfaces so that $n_{1}=n_{L_{S}}\left(P_{1}=-P_{2}<0\right.$ for hole accumulation). For the same amount of injected charge $n$, the accumulation of carriers on both contacts does not provide significant charge redistribution and leads to a decrease of the maximal achievable charge density in the boundary planes $n_{1}(\max )=n_{L_{S}}(\max )=L_{S} n / 2$, as compared to the state with the parallel $P$ where the maximal possible accumulation density is $n_{1}(\max )=L_{S} n$. Consequently, the antiparallel polarization results in lower $T_{c}$ (see Fig. 1(b), where $P$ directed towards the interfaces corresponds to electron accumulation). In particular, for $L_{S}=2$, the antiparallel polarization does not give the re- distribution of the injected charge $\left(n_{1}=n_{2}=n\right)$ which results in the constant $T_{c}$ in Fig. 11(b).

Based on these advantages of sandwiches, we propose that the operation of an SuFET containing an S-film confined between two FE-layers, can be realized in two steps which are shown in two possible realizations in Fig. 2(a) and (b). Here, step(1) switches the SuFET to the superconducting state with the enhanced $T_{c}$ caused by the parallel polarization in the FE-gates. This can be achieved by applying the voltages $V_{g}$ to the gate electrode $\mathrm{FE}_{1}$ and $-V_{g}$ to $\mathrm{FE}_{2}$. In the first realization of $\operatorname{step}(1)$ (Fig. 2(a)), the power supply simultaneously moves the opposite charge to the gate electrodes. To reset the SuFET to the state with lower $T_{c}(\operatorname{step}(2))$, one destroys the accumulation layer at the $\mathrm{FE}_{1}-\mathrm{S}$ contact, which is realized here by decreasing the gate voltages $V_{g}$ and tuning the gate polarization to zero. However, with the gates fabricated from the PZT(BTO)-compounds, a nonzero spontaneous polarization $P_{s} \neq 0$ at $V_{g}=0$ could result only in a partially removed accumulation region. Thus, in this realization we propose to use the gates made with STO or BSTO with high $\mathrm{Sr}$ content, so that $P_{s} \approx 0$ at temperatures close to $T_{c}$. The corresponding modulation of $T_{c}$ is illustrated in Fig. 31(a) for the $s$-and $d$-wave pairing in the superconducting channel. As compared to the FE-S-bilayers and s-symmetry channels, the remarkable shift of $T_{c}$ in step(1) is obtained for the $d$-wave sandwiches, which strongly supports the use of high- $T_{c}$ cuprates in the proposed SuFETs. For a more realistic analysis of $d$-wave pairing, we choose the band structure with the next-nearest-neighbor hopping which resembles the Fermi surface of $\mathrm{YBa}_{2} \mathrm{Cu}_{3} \mathrm{O}_{7-\delta}$. The electron density is taken near half-filling, where the cases $n=0.9$ (hole density $x=1-n=0.1)$ and $n=0.75(x=0.25)$ should correspond to the under- and overdoped regions. There are a few important points to note. First, for underdoping, the obtained increase of $T_{c}$ is stronger than that for overdoping, which agrees well with the recent observations of the strong field-effect in the underdoped region of the cuprate phase diagram 2]. Second, as the present studies are based on a BCS-type model, the obtained here estimates for the $T_{c}$ increase in the underdoped region consider only the mean-field boundary for the transition temperature. To get more precise estimates for underdoping, the lowering of $T_{c}$ by phase fluctuations 4 ] should be analyzed, which is the object of future studies.

In the second realization (Fig. 2(b)), the electric power sweeps the charge from the $\mathrm{FE}_{1}$-gate electrode and injects it into the $\mathrm{S}$-channel. Here, the $\mathrm{FE}_{2}$-polarization is used to control the additional enhancement of $T_{c}$ in the channel. Reversing the $\mathrm{FE}_{2}$-polarization switches the SuFET into the antiparallel polarization state with the lower $T_{c}$. The modulation of $T_{c}$ by a change of the $\mathrm{FE}_{2}$-gate field for the fixed $\mathrm{FE}_{1}$-gate field is shown in Fig. [3(b), where the strong increase of $T_{c}$ in a $d$-wave conducting channel is obtained when going from the step 
(2) to the step (1) in Fig. 2(b). Another possible modification of the second realization could include the injection of additional charge from the $\mathrm{FE}_{2}$-gate electrode into the channel and thus achievement of higher $n$ (or $x$ ) in the state with antiparallel polarization. Although the charge densities in both accumulation planes in this case are comparable to those with the parallel $P$, the question of the inter-planar charge modulation and thickness dependence of $T_{c}$ needs further theoretical studies.

In conclusion, we have discussed the schemes of SuFETs based on ferroelectric-superconducting sandwiches, where the $d$-wave channels show great potential for strong modulation of $T_{c}$. However, for a successful implementation, important theoretical and technological issues (related to the the growth of good quality interfaces where the interface steps do not significantly affect the charge redistribution in S-film) need to be solved.

This work was supported by the DFG Grants No. SPP1056, SFB-484 and the BMBF Grant(13N6918A).

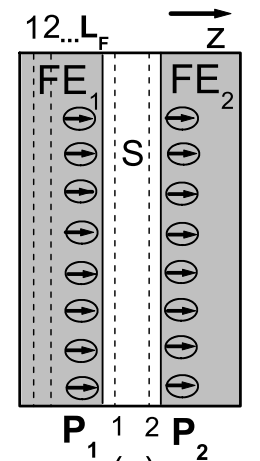

(a)

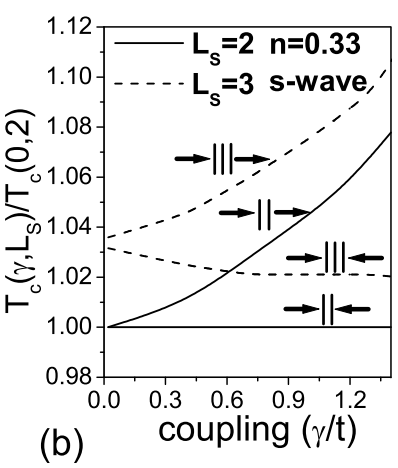

(b)
FIG. 1: (a) Scheme of FE-S-FE sandwich with the uniform parallel spontaneous polarization in FE-layers. (b) $T_{c}$ vs $\gamma$ in sandwiches containing $L_{F}=10 \mathrm{FE}$-monolayers, where the cases of parallel and antiparallel gate polarization are shown for a comparison. Here $V^{0} / t=-3.5, J_{F} / t=1, t_{\perp} / t=0.1$, and the electronic band filling $n=0.3$. All the temperatures are scaled by $T_{c}\left(\gamma=0, L_{S}=2\right)$, and we show here the case of $s$-wave pairing in S-film.

[*] Electronic mail: pavlenko@mailaps.org

[1] C.H. Ahn, J.-M. Triscone, and J. Mannhart, Nature 424, 1015 (2003); J. Mannhart, Supercond. Sci. Technol. 9, 49 (1996).

[2] D. Matthey, S. Gariglio, C.H. Ahn, J.-M. Triscone, Physica C 372-376, 583 (2002).

[3] X.X. Xi, C. Doughty, A. Walkenhorst, C. Kwon, Q. Li, and T. Venkatesan, Phys. Rev. Lett. 68, 1240 (1992); C.H. Ahn, S. Gariglio, P. Paruch, T. Tybell, L. Antognazza, J.-M. Triscone, Science 284, 1152 (1999).

[4] V.J. Emery and S.A. Kivelson, Phys. Phys. Lett. 74, 3253 (1995).

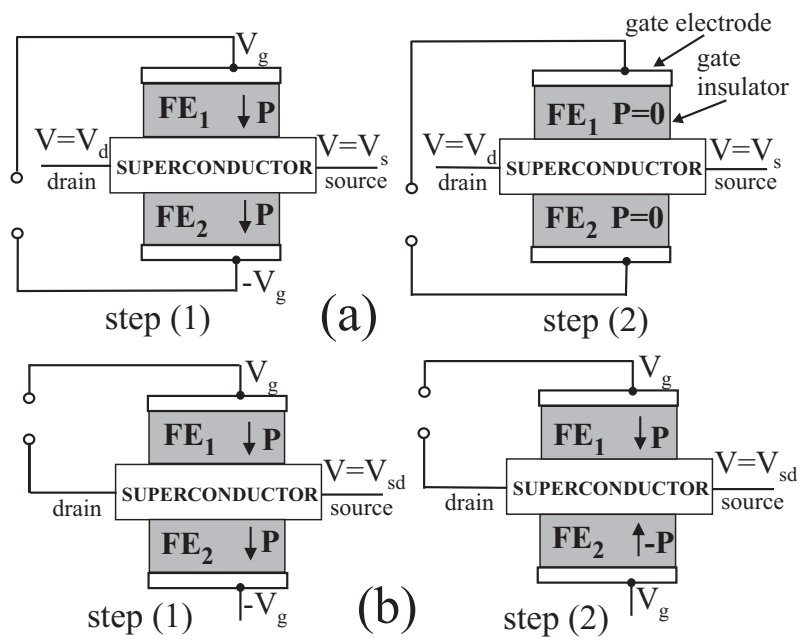

FIG. 2: Proposed schemes of SuFET based on confined FES-FE geometry. In step(1) with parallel gate polarization the SuFET is in the state with enhanced $T_{c}$, whereas $\operatorname{step}(2)$ destroys (a) or decreases (b) the electron accumulation at $\mathrm{FE}_{1}-\mathrm{S}$ interface and thus switches SuFET into the state with low $T_{c}$.

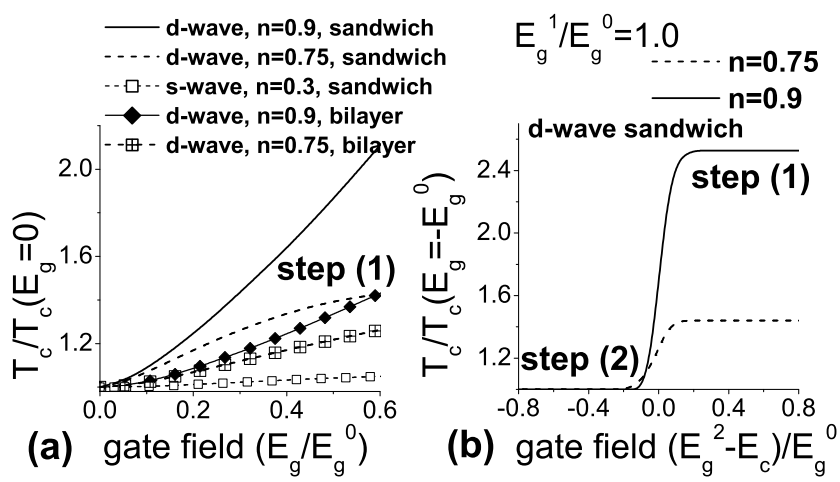

FIG. 3: Superconducting transition temperature under variation of gate electric fields (a) for scheme shown in Fig. 22 (a) where the gate fields $E_{g}^{1}=E_{g}^{2}=E_{g}$ set the parallel polarization in both STO-gates of thickness $d=300 \mathrm{~nm}$ with the dielectric constant $\varepsilon=100$ and maximal achievable polarization $P_{\max }=5 \mu \mathrm{C} \mathrm{cm}^{-2}$; (b) for scheme shown in Fig. 2 (b) where the $\mathrm{FE}_{1}$-gate field is keeping fixed whereas the $\mathrm{FE}_{2}$-gate field is switched resulting in a reverse of the $\mathrm{FE}_{2}$-polarization at the coercive field $E_{c}$ in step (2). Here $\gamma / t=0.5$ and FE-layers with a polarization $P_{S}=30 \mu \mathrm{C} \mathrm{cm}^{-2}$ are considered). In S-film for $d$-wave pairing, the planar band structure $\varepsilon_{\boldsymbol{k}}=-2 t\left(\cos k_{x}+\cos k_{y}\right)-4 t_{2} \cos k_{x} \cos k_{y}$ is chosen with next-nearest-neighbor hopping $t_{2} / t=-0.4, t_{\perp} / t=0.05$ and $V^{0} / t=-0.5$. All gate fields are scaled by a characteristic maximum field $E_{g}^{0} \approx 10^{6} \mathrm{~V} / \mathrm{cm}$.

[5] G.Yu. Logvenov, A. Sawa, C.W. Schneider, and J. Mannhart, Appl. Phys. Lett. 83, 3528 (2003).

[6] N. Pavlenko, Phys. Rev. B 70, 094519 (2004).

[7] M.E. Lines, A.M. Glass, Principles and Applications of Ferroelectrics and Related Materials (Clarendon Press, Oxford, 1977).

[8] J. Junquera and P. Ghosez, Nature 422, 506 (2003).

[9] N. Pavlenko and F. Schwabl, Phys. Rev. B 67, 094516 
(2003).

[10] N. Pavlenko and F. Schwabl, Appl. Phys. A in press;
N. Pavlenko, Ann. Phys.-Leipzig 13, 52 (2004). 